\title{
Weisskopf-Wigner Decay Theory for the Energy-Driven Stochastic Schrödinger Equation
}

\author{
Stephen L. Adler円 \\ Institute for Advanced Study \\ Einstein Drive \\ Princeton, NJ 08540
}

(Dated: September, 2002)

\begin{abstract}
We generalize the Weisskopf-Wigner theory for the line shape and transition rates of decaying states to the case of the energy-driven stochastic Schrödinger equation that has been used as a phenomenology for state vector reduction. Within the standard approximations used in the Weisskopf-Wigner analysis, and assuming that the perturbing potential inducing the decay has vanishing matrix elements within the degenerate manifold containing the decaying state, the stochastic Schrödinger equation linearizes. Solving the linearized equations, we find no change from the standard analysis in the line shape or the transition rate per unit time. The only effect of the stochastic terms is to alter the early time transient behavior of the decay, in a way that eliminates the quantum Zeno effect. We apply our results to estimate experimental bounds on the parameter governing the stochastic effects. In an Added Note, elegant stochastic-theoretic methods suggested by Diósi are used to rederive the principal results, without the assumptions needed to linearize the stochastic equation, and to give analogous results for the Rabi oscillations of a two-level system.
\end{abstract}

*adler@ias.edu 


\section{INTRODUCTION}

There has recently been considerable interest in the possibility that quantum mechanics, and the Schrödinger equation, may be modified at a very low level by effects arising from Planck scale physics. Such speculations have been motivated on the one hand by considerations from string theory [1] and quantum gravity [2], and on the other hand by efforts [2, 3, 4] to achieve an objective equation describing state vector reduction. The majority of the objective reduction discussions fall into two classes: those that postulate a stochastic process producing spatial localization [3], and those that postulate an analogous stochastic process leading to localization in energy [4] (the so-called "energy-driven" stochastic Schrödinger equation.) Both the spatial localization and the energy localization stochastic Schrödinger equations avoid problems with superluminal signal propagation that characterize attempts at deterministic nonlinear modifications of the Schrödinger equation [5]. We find the energy-driven approach particularly appealing because it is energy conserving, leads with no approximations to Born rule probabilities and to the Lüders projection postulate, has sensible clustering properties, and when environmental interactions are taken into account explains state vector reduction with a single Planck scale stochastic parameter [4, 6.

Although physical prejudices might suggest a Planck scale magnitude for the stochastic parameter in the energy-driven equation, one can instead take the point of view that the stochastic parameter can have a priori any value, and use current experimental information to place bounds on it. This approach has been pursued [7] in the context of particle physics systems that exhibit oscillations between different mass eigenstates (the $K$-meson, $B$-meson, and neutrino systems), with results that are summarized in the final section of this paper. An alternative source of bounds on the stochastic parameter could come from experiments observing decays and line shapes in atomic and particle systems, since if the stochastic terms in the Schrödinger equation were to change the standard Weisskopf-Wigner analysis of decay processes in a significant way, then observable effects could result. Thus, to pursue phenomenological studies of the energy-driven equation, it is important to generalize the standard Weisskopf-Wigner decay theory [8] to include effects of the energy-driven stochastic terms. This is the problem that is analyzed in this paper. 


\section{THE ENERGY-DRIVEN STOCHASTIC SCHRÖDINGER EQUATION AND PROPERTIES OF THE ITÔ STOCHASTIC CALCULUS}

Letting $|\psi\rangle$ denote a unit normalized Schrödinger picture state vector, the standard form [3, [4, 6] of the energy-driven stochastic Schrödinger equation is (with $\hbar=1$ )

$$
d|\psi\rangle=-i H|\psi\rangle d t-\frac{1}{8} \sigma^{2}(H-\langle H\rangle)^{2}|\psi\rangle d t+\frac{1}{2} \sigma(H-\langle H\rangle)|\psi\rangle d W_{t}
$$

Here $H$ is the Hamiltonian, $\langle H\rangle=\langle\psi|H| \psi\rangle$ is the expectation of the Hamiltonian in the state $|\psi\rangle, \sigma$ is a numerical parameter governing the strength of the stochasticity, and $d W_{t}$ is an Itô stochastic differential that, together with $d t$, obeys the standard Itô calculus rules [9]

$$
d W_{t}^{2}=d t, \quad d W_{t} d t=d t^{2}=0 .
$$

By construction, the nonlinear evolution of Eq. (15) guarantees the preservation in time of the unit normalization of the state vector $|\psi\rangle$.

In the following sections, we shall need a number of properties of the Itô calculus that we summarize here. First of all, in the Itô calculus the Leibnitz chain rule generalizes to

$$
d(A B)=(A+d A)(B+d B)-A B=(d A) B+A d B+d A d B \quad,
$$

with the final term $d A d B$ contributing a term proportional to $d t$ when the $d W_{t}$ contributions to both $d A$ and $d B$ are nonzero. Letting $W_{t}$ be the Brownian motion

$$
W_{t}=\int_{0}^{t} d W_{u}
$$

we see in particular that

$$
d \exp \left(\alpha W_{t}\right)=\exp \left(\alpha W_{t}\right)\left[\exp \left(\alpha d W_{t}\right)-1\right]=\exp \left(\alpha W_{t}\right)\left[\alpha d W_{t}+\frac{1}{2} \alpha^{2} d t\right] .
$$

Letting $E[\ldots]$ denote the stochastic expectation of its argument, and letting $A(t)$ denote any function of the stochastic process up to time $t$, we have

$$
E\left[d W_{t} A(t)\right]=0
$$

since the Itô differential refers to the time interval from $t$ to $t+d t$, and hence is statistically independent of the process up to time $t$. Thus, taking the expectation of Eq. (2C), we get the differential equation

$$
d E\left[\exp \left(\alpha W_{t}\right)\right]=E\left[\exp \left(\alpha W_{t}\right)\right] \frac{1}{2} \alpha^{2} d t
$$


which can be immediately integrated to give

$$
E\left[\exp \left(\alpha W_{t}\right)\right]=\exp \left(\frac{1}{2} \alpha^{2} t\right)
$$

a result that will be needed later on.

Let us make an elementary application of the Itô formalism, to write the stochastic Schrödinger equation of Eq. (1a) in an equivalent form. First of all, forming the density matrix

$$
\rho=|\psi\rangle\langle\psi|
$$

we have from Eq. (2a),

$$
d \rho=(d|\psi\rangle)\langle\psi|+| \psi\rangle d\langle\psi|+d| \psi\rangle d\langle\psi|
$$

which on substitution of Eq. (1a) and use of the Itô calculus rules of Eq. (1b) gives the evolution equation for the density matrix,

$$
d \rho=i[\rho, H] d t-\frac{1}{8} \sigma^{2}[H,[H, \rho]] d t+\frac{1}{2} \sigma[\rho,[\rho, H]] d W_{t}
$$

Taking the stochastic expectation of this equation, using Eq. (3a), gives a differential equation of the Lindblad type [10] for $E[\rho]$,

$$
\frac{d E[\rho]}{d t}=i[E[\rho], H]-\frac{1}{8} \sigma^{2}[H,[H, E[\rho]]] .
$$

The fact that this equation is linear (in contrast to Eq. (4c), which is nonlinear) is the fundamental reason [5] why Eq. (1a) does not give rise to superluminal signal propagation.

\section{INITIAL FORMULATION OF THE DECAY PROBLEM}

Let us now formulate the decay problem for the stochastic Schrödinger equation of Eq. (1a), following the standard procedure for the usual Schrödinger equation without stochastic terms. We suppose that for times $t \leq 0$ the Hamiltonian $H$ is given by an unperturbed Hamiltonian $H_{0}$, with eigenstates $|n\rangle$ and eigenvalues $E_{n}$,

$$
H_{0}|n\rangle=E_{n}|n\rangle,
$$

and that the system under consideration is in an eigenstate $\left|s_{A}\right\rangle$ with eigenvalue $E_{s}$, which is one of a set of degenerate energy eigenstates $\left|s_{a}\right\rangle, a=1, \ldots, D$. Because Eq. (1a) 
acts as an ordinary Schrödinger evolution on a state $|\psi\rangle$ that is an energy eigenstate, the system remains in the state $\left|s_{A}\right\rangle$ as long as the Hamiltonian remains equal to $H_{0}$. Hence the starting point for the standard decay analysis [11] is also a consistent starting point for its stochastic extension under Eq. (10). As in the standard procedure, we assume that at $t=0$ a time-independent perturbation $V$ is switched on, so that for times $t>0$ the Hamiltonian is $H=H_{0}+V$. The initial state $\left|s_{A}\right\rangle$ is then no longer an energy eigenstate, and so will decay into various other states $|m\rangle$; our problem, as in the usual case, is to find the partial transition rates for this decay and the probability amplitude for the system to remain in the initial degenerate group of states.

In formulating this problem, it is convenient to expand the state $|\psi\rangle$ over the basis $|n\rangle$ and, as in the standard case, to remove the Schrödinger time evolution associated with the unperturbed Hamiltonian $H_{0}$, by writing

$$
|\psi(t)\rangle=\sum_{n}|n\rangle \exp \left(-i E_{n} t\right) C_{n}(t)
$$

Substituting Eq. (6b) into Eq. (1a), and projecting on $\langle m|$, it is a matter of straightforward but somewhat tedious algebra to compute the stochastic evolution equation for $C_{m}(t)$, with the result

$$
\begin{aligned}
d C_{m}(t) & =\alpha_{m} C_{m}(t)+\sum_{n} \beta_{m n} C_{n}(t) \\
\alpha_{m} & =-\frac{1}{8} \sigma^{2}\left(E_{m}-\langle H\rangle\right)^{2} d t+\frac{1}{2} \sigma\left(E_{m}-\langle H\rangle\right) d W_{t} \\
\beta_{m n} & =-i V_{m n} \exp \left[i\left(E_{m}-E_{n}\right) t\right] d t-\frac{1}{8} \sigma^{2}\left[\left(E_{m}+E_{n}-2\langle H\rangle\right) V_{m n}+\left(V^{2}\right)_{m n}\right] \exp \left[i\left(E_{m}-E_{n}\right) t\right] d t \\
& +\frac{1}{2} \sigma V_{m n} \exp \left[i\left(E_{m}-E_{n}\right) t\right] d W_{t} .
\end{aligned}
$$

The corresponding expression for $\langle H\rangle$ is

$$
\langle H\rangle=\sum_{n} E_{n}\left|C_{n}(t)\right|^{2}+\sum_{m n} V_{m n} \exp \left[i\left(E_{m}-E_{n}\right) t\right] C_{m}^{*}(t) C_{n}(t) .
$$

In these equations $V_{m n}$ and $\left(V^{2}\right)_{m n}$ denote the respective matrix elements

$$
V_{m n}=\langle m|V| n\rangle, \quad\left(V^{2}\right)_{m n}=\left\langle m\left|V^{2}\right| n\right\rangle .
$$

\section{APPROXIMATION TO LEADING ORDER IN $V$}

Equations (7a-c) are a complicated, nonlinear set of stochastic differential equations, and so to solve them approximations will be needed. Following the Weisskopf-Wigner analysis, 
we shall make the approximation of regarding $V$ as a small perturbation. The coefficients $C_{m}, m \notin\left\{s_{a}\right\}$ for states not in the initial degenerate manifold will then be of order $O(V)$, and we neglect $O\left(V^{2}\right)$ and higher contributions to them (except those arising implicitly through our solution for the $C_{s_{a}}$ ). On the other hand, the coefficients $C_{s_{a}}$ of states in the degenerate manifold can be of order unity, and we calculate these coefficients to order $V^{2}$ accuracy, neglecting corrections of order $V^{3}$ and higher. In a similar fashion, in expressions involving the stochasticity parameter $\sigma$, we shall retain terms of order $\sigma V$ and its powers $(\sigma V)^{2}$, etc., but shall neglect terms of order $\sigma V^{2}$ and higher that involve extra factors of $V$ relative to the terms that we are retaining. Finally, although we shall see that $E_{m}-E_{s}$ is effectively small, we shall retain all terms of order $\sigma\left(E_{m}-E_{s}\right), \sigma^{2}\left(E_{m}-E_{s}\right),\left[\sigma\left(E_{m}-E_{s}\right)\right]^{2}$, etc., but shall drop terms $\sigma\left(E_{m}-E_{s}\right) \sigma O\left(V^{2}\right)$ that are smaller than these by a factor of order $\sigma V^{2}$ or $V^{2}$.

Making use of the perturbative ordering of the coefficients $C_{n}$, we begin by simplifying and approximating the expression in Eq. (7b) for $\langle H\rangle$. Separating off the states in the initial degenerate manifold, the sum in Eq. (7b) becomes

$$
\langle H\rangle=E_{s} \sum_{a}\left|C_{s_{a}}\right|^{2}+\sum_{a b} V_{s_{a} s_{b}} C_{s_{a}}^{*} C_{s_{b}}+O\left(V^{2}\right) .
$$

However, since the state vector $|\psi\rangle$ remains unit normalized, we have

$$
\sum_{a}\left|C_{s_{a}}\right|^{2}=1-\sum_{m \notin\left\{s_{a}\right\}}\left|C_{m}\right|^{2}=1+O\left(V^{2}\right),
$$

and so we have

$$
\langle H\rangle=E_{s}+\sum_{a b} V_{s_{a} s_{b}} C_{s_{a}}^{*} C_{s_{b}}+O\left(V^{2}\right) .
$$

If we substitute Eq. (80) back into Eq. (7a), we are still left with a nonlinear set of equations. Therefore we shall also introduce the simplifying assumption that the perturbing potential has vanishing matrix elements within the degenerate manifold containing the initial state, so that

$$
V_{s_{a} s_{b}}=0, \quad a, b=1, \ldots, D .
$$

There are important, physically relevant cases in which Eq. (9a) is obeyed as a result of selection rules. For example, for radiative decays treated in the electric dipole approximation, with $H_{0}$ taken as the atomic Hamiltonian plus the free radiation Hamiltonian, and with $V$ taken as the atomic coupling to the transverse electromagnetic modes, Eq. (9a) is obeyed as 
a result of parity invariance when the states in the initial degenerate manifold all have the same parity. (We caution, however, that Eq. (9a) is not valid for the analysis of $K$ (or $B$ ) meson systems when $H_{0}$ is taken as the strong interaction Hamiltonian. Thus here either one has to employ the nonlinear equations following from Eq. (80), or one has to redefine $H_{0}$ so as to impose Eq. (9a) by including in $H_{0}$ the $|\Delta S|=2$ (or $|\Delta C|=2$ ) weak interaction effective Hamiltonian terms, with $V$ defined to contain only the $|\Delta S|=1$ (or $|\Delta C|=1$ ) weak interaction terms responsible for $K$ (or $B$ ) meson decays. Such a redefinition is consistent in the vacuum saturation approximation for the $|\Delta S|=2$ (or $|\Delta C|=2$ ) terms.)

With the simplifying assumption of Eq. (9a), Eq. (8c) becomes simply

$$
\langle H\rangle=E_{s}+O\left(V^{2}\right)
$$

Substituting this into Eq. (7a), and dropping terms that are not of leading order in $V$ in the sense defined above, Eqs. (7a,7b) simplify to the following set of linear equations,

$$
\begin{aligned}
d C_{m}(t) & =\left(\alpha_{m}^{(1)} d W_{t}+\alpha_{m}^{(2)} d t\right) C_{m}(t)+\sum_{n} \exp \left[i\left(E_{m}-E_{n}\right) t\right]\left(\gamma_{m n}^{(1)} d W_{t}+\gamma_{m n}^{(2)} d t\right) C_{n}(t) \\
\alpha_{m}^{(1)} & =\frac{1}{2} \sigma\left(E_{m}-E_{s}\right), \quad \alpha_{m}^{(2)}=-\frac{1}{8} \sigma^{2}\left(E_{m}-E_{s}\right)^{2}=-\frac{1}{2}\left(\alpha_{m}^{(1)}\right)^{2} \\
\gamma_{m n}^{(1)} & =\frac{1}{2} \sigma V_{m n}, \quad \gamma_{m n}^{(2)}=-i V_{m n}-\frac{1}{8} \sigma^{2}\left[\left(E_{m}+E_{n}-2 E_{s}\right) V_{m n}+\left(V^{2}\right)_{m n}\right]
\end{aligned}
$$

Corresponding to the magnitude ordering of the coefficients $C_{m}$ introduced above, it is convenient to rewrite Eq. (10a) as separate equations for the two cases, $m \in\left\{s_{a}\right\}$ and $m \notin\left\{s_{a}\right\}$. For $m \in\left\{s_{a}\right\}$ the coefficients $\alpha_{s}^{(1,2)}$ vanish; separating the sum over $n$ into terms where $n \in\left\{s_{a}\right\}$ and $n \notin\left\{s_{a}\right\}$, using the assumption of Eq. (9a), and dropping terms of nonleading order in $V$, we get

$$
\begin{aligned}
d C_{s_{a}}(t) & =-\frac{1}{8} \sigma^{2} d t \sum_{b}\left(V^{2}\right)_{s_{a} s_{b}} C_{s_{b}}(t) \\
& +\sum_{n \notin\left\{s_{a}\right\}} \exp \left[i\left(E_{s}-E_{n}\right) t\right]\left(\gamma_{s_{a} n}^{(1)} d W_{t}+\gamma_{s_{a} n}^{(2)} d t\right) C_{n}(t) \\
\gamma_{s_{a} n}^{(1)} & =\frac{1}{2} \sigma V_{s_{a} n}, \quad \gamma_{s_{a} n}^{(2)} \simeq-i V_{s_{a} n} f_{n}
\end{aligned}
$$

where we have introduced the definition

$$
f_{n}=1-\frac{i}{8} \sigma^{2}\left(E_{n}-E_{s}\right)
$$


For $m \notin\left\{s_{a}\right\}$ the coefficients $\alpha_{m}^{(1,2)}$ are nonzero, but only the terms with $n \in\left\{s_{a}\right\}$ have to be retained in the sum over $n$, and so we similarly get

$$
\begin{aligned}
d C_{m}(t) & =\left(\alpha_{m}^{(1)} d W_{t}+\alpha_{m}^{(2)} d t\right) C_{m}(t) \\
& +\exp \left[i\left(E_{m}-E_{s}\right) t\right] \sum_{a}\left(\gamma_{m s_{a}}^{(1)} d W_{t}+\gamma_{m s_{a}}^{(2)} d t\right) C_{s_{a}}(t) \\
\gamma_{m s_{a}}^{(1)} & =\frac{1}{2} \sigma V_{m s_{a}}, \quad \gamma_{m s_{a}}^{(2)} \simeq-i V_{m s_{a}} f_{m} .
\end{aligned}
$$

Equations (10a-d) are the basic system of stochastic differential equations that we shall solve in the subsequent sections.

\section{EQUATIONS FOR EXPECTATIONS OF THE COEFFICIENTS}

The principal quantities that we wish to calculate are the expectations $E\left[\left|C_{m}(t)\right|^{2}\right]$ of the squared magnitudes of the coefficients, since these give the expectations of the probabilities for the various states to be occupied. We shall show in this section that, within our approximations, these can be directly related to the expectations $E\left[C_{m}(t)\right]$ of the coefficients themselves, for which we shall derive a closed, linear set of ordinary differential equations.

Again, we consider separately the cases $m \in\left\{s_{a}\right\}$ and $m \notin\left\{s_{a}\right\}$. For $C_{s_{a}}$, we write

$$
C_{s_{a}}(t)=E\left[C_{s_{a}}(t)\right]+\Delta_{a}(t)
$$

with $E\left[\Delta_{a}(t)\right]=0$, and with $\Delta_{a}(0)=0$ since the stochastic terms in the differential equation act only after $t=0$. However, referring to Eq. (10b) we see that $d C_{s_{a}}$ is of order $V^{2}$, and so $\Delta_{a}(t)$ must also be of order $V^{2}$. Therefore

$$
E\left[\left|C_{s_{a}}(t)\right|^{2}\right]=\left|E\left[C_{s_{a}}(t)\right]\right|^{2}+O\left(V^{4}\right),
$$

and so to the accuracy to which we are working, we can compute $E\left[\left|C_{s_{a}}(t)\right|^{2}\right]$ from the expectation $E\left[C_{s_{a}}(t)\right]$, ignoring the effects of fluctuations.

We consider next $E\left[\left|C_{m}(t)\right|^{2}\right]$ for $m \notin\left\{s_{a}\right\}$. Applying the Itô rule of Eq. (2a), we have

$$
d E\left[\left|C_{m}(t)\right|^{2}\right]=E\left[\left(d C_{m}^{*}(t)\right) C_{m}(t)+C_{m}^{*}(t) d C_{m}(t)+d C_{m}^{*}(t) d C_{m}(t)\right] .
$$

Substituting Eq. (10d) for $d C_{m}(t)$ and using Eq. (3a), which eliminates the $d W_{t}$ contributions, and using the fact that to leading order in $V$ we can replace $C_{s_{a}}(t)$ by its expectation, 
we get after some algebraic simplification the formula

$$
\begin{aligned}
\frac{d}{d t} E\left[\left|C_{m}(t)\right|^{2}\right] & =\exp \left[-i\left(E_{m}-E_{s}\right) t\right] i f_{m} E\left[C_{m}(t)\right] \sum_{a} V_{m s_{a}}^{*} E\left[C_{s_{a}}^{*}(t)\right] \\
& -\exp \left[i\left(E_{m}-E_{s}\right) t\right] i f_{m}^{*} E\left[C_{m}^{*}(t)\right] \sum_{a} V_{m s_{a}} E\left[C_{s_{a}}(t)\right] \\
& +\frac{1}{4} \sigma^{2}\left|\sum_{a} V_{m s_{a}} E\left[C_{s_{a}}(t)\right]\right|^{2},
\end{aligned}
$$

which can be integrated to give $E\left[\left|C_{m}(t)\right|^{2}\right]$ once the expectations $E\left[C_{m}(t)\right]$ and $E\left[C_{s_{a}}(t)\right]$ are known.

To get a closed set of equations for the expectations of the coefficients, we simply take the expectations of Eqs. (10b) and (10d), and use Eq. (3a), which again eliminates the $d W_{t}$ contributions. For $E\left[C_{s_{a}}\right]$ we thus get

$$
\begin{aligned}
\frac{d}{d t} E\left[C_{s_{a}}(t)\right] & =-\frac{1}{8} \sigma^{2} \sum_{b}\left(V^{2}\right)_{s_{a} s_{b}} E\left[C_{s_{b}}(t)\right] \\
& +\sum_{n \notin\left\{s_{a}\right\}} \exp \left[i\left(E_{s}-E_{n}\right) t\right](-i) V_{s_{a} n} f_{n} E\left[C_{n}(t)\right]
\end{aligned}
$$

while for $E\left[C_{m}(t)\right]$ with $m \notin\left\{s_{a}\right\}$ we find

$$
\begin{aligned}
\frac{d}{d t} E\left[C_{m}(t)\right] & \left.=-\frac{1}{8} \sigma^{2}\left(E_{m}-E_{s}\right)^{2}\right) E\left[C_{m}(t)\right] \\
& +\exp \left[i\left(E_{m}-E_{s}\right) t\right] \sum_{b}(-i) V_{m s_{b}} f_{m} E\left[C_{s_{b}}(t)\right] .
\end{aligned}
$$

\section{SOLUTIONS FOR EXPECTATIONS OF THE COEFFICIENTS}

We proceed now to solve the linear system of equations for the expectations of the coefficients given in Eqs. (13a,b). Since the problem is defined on the half line $t>0$, the natural way to do this is by using the Laplace transform. Defining

$$
g_{m}(p)=\int_{0}^{\infty} d t \exp (-p t) E\left[C_{m}(t)\right]
$$

we have, by an integration by parts,

$$
\int_{0}^{\infty} d t \exp (-p t) \frac{d E\left[C_{m}(t)\right]}{d t}=p g_{m}(p)-E\left[C_{m}(0)\right]
$$

and also

$$
\int_{0}^{\infty} d t \exp (-p t) \exp \left[i\left(E_{m}-E_{n}\right) t\right] E\left[C_{n}(t)\right]=g_{n}\left(p-i E_{m}+i E_{n}\right)
$$


with the integrals in Eqs. (14a-c) defining analytic functions of $p$ in the right hand half plane Re $p>0$. The inversion of the Laplace transform is given by the formula

$$
E\left[C_{m}(t)\right]=\frac{1}{2 \pi i} \int_{\epsilon-i \infty}^{\epsilon+i \infty} d p \exp (p t) g_{m}(p)
$$

with $\epsilon>0$ an infinitesimal positive constant.

Taking the Laplace transform of Eqs. (13a,b), and using the initial conditions $E\left[C_{s_{a}}(0)\right]=$ $C_{s_{a}}(0)=\delta_{a A}$ and $E\left[C_{m}(0)\right]=C_{m}(0)=0, m \notin\left\{s_{a}\right\}$, we get

$$
\begin{aligned}
p g_{s_{a}}(p)-\delta_{a A} & =-\frac{1}{8} \sigma^{2} \sum_{b}\left(V^{2}\right)_{s_{a} s_{b}} g_{s_{b}}(p) \\
& +\sum_{n \notin\left\{s_{a}\right\}}(-i) V_{s_{a} n} f_{n} g_{n}\left(p+i E_{n}-i E_{s}\right)
\end{aligned}
$$

and for $m \notin\left\{s_{a}\right\}$,

$$
\begin{aligned}
p g_{m}(p) & \left.=-\frac{1}{8} \sigma^{2}\left(E_{m}-E_{s}\right)^{2}\right) g_{m}(p) \\
& +\sum_{b}(-i) V_{m s_{b}} f_{m} g_{s_{b}}\left(p+i E_{s}-i E_{m}\right) .
\end{aligned}
$$

Solving Eq. (15b) for $g_{m}(p), m \notin\left\{s_{a}\right\}$, and shifting $p \rightarrow p+i E_{m}$ in the solution, we get

$$
g_{m}\left(p+i E_{m}\right)=\left[p+i E_{m}+\frac{1}{8} \sigma^{2}\left(E_{m}-E_{s}\right)^{2}\right]^{-1} \sum_{b}(-i) V_{m s_{b}} f_{m} g_{s_{b}}\left(p+i E_{s}\right) \text {. }
$$

Shifting $p \rightarrow p+i E_{s}$ in Eq. (15a), and then substituting Eq. (16a), we get an algebraic equation for the set of quantities $g_{s_{b}}\left(p+i E_{s}\right)$,

$$
\begin{aligned}
& \sum_{b} K_{a b} g_{s_{b}}\left(p+i E_{s}\right)=\delta_{a A} \\
& K_{a b}=\left(p+i E_{s}\right) \delta_{a b}+\frac{1}{8} \sigma^{2}\left(V^{2}\right)_{s_{a} s_{b}}+\sum_{m \notin\left\{s_{a}\right\}} \frac{f_{m}^{2} V_{s_{a} m} V_{m s_{b}}}{p+i E_{s}+i\left(E_{m}-E_{s}\right) f_{m}}
\end{aligned}
$$

In physically interpreting these equations, we must remember that the Laplace transform variable $p$ is related to the usual energy variable $E$ by $p=-i E$. Making this substitution in Eqs. (16a,b) we have respectively

$$
g_{m}\left(-i E+i E_{m}\right)=\left[-i E+i E_{m}+\frac{1}{8} \sigma^{2}\left(E_{m}-E_{s}\right)^{2}\right]^{-1} \sum_{b}(-i) V_{m s_{b}} f_{m} g_{s_{b}}\left(-i E+i E_{s}\right)
$$

and

$$
\begin{aligned}
& \sum_{b} K_{a b} g_{s_{b}}\left(-i E+i E_{s}\right)=\delta_{a A} \\
& K_{a b}=\left(-i E+i E_{s}\right) \delta_{a b}+\frac{1}{8} \sigma^{2}\left(V^{2}\right)_{s_{a} s_{b}}+\sum_{m \notin\left\{s_{a}\right\}} \frac{f_{m}^{2} V_{s_{a} m} V_{m s_{b}}}{-i E+i E_{s}+i\left(E_{m}-E_{s}\right) f_{m}}
\end{aligned}
$$


Corresponding to the changes of variable that have been made, the inversion formulas become

$$
\begin{aligned}
E\left[C_{s_{a}}(t)\right] & =\frac{1}{2 \pi} \exp \left(i E_{s} t\right) \int_{i \epsilon-\infty}^{i \epsilon+\infty} d E \exp (-i E t) g_{s_{a}}\left(-i E+i E_{s}\right) \\
E\left[C_{m \notin\left\{s_{a}\right\}}(t)\right] & =\frac{1}{2 \pi} \exp \left(i E_{m} t\right) \int_{i \epsilon-\infty}^{i \epsilon+\infty} d E \exp (-i E t) g_{m}\left(-i E+i E_{m}\right)
\end{aligned}
$$

Inspecting the equation for the kernel $K_{a b}$, we see that apart from order $V^{2}$ terms it is a diagonal matrix $\left(-i E+i E_{s}\right) \delta_{a b}$. Hence the solution $g_{s_{b}}\left(-i E+i E_{s}\right)$, on the inversion contour of integration, will be appreciable only in the vicinity of $E=i \epsilon+E_{s}$, that is, only near energy shell. This motivates the Weisskopf-Wigner approximation of replacing $E$ in the denominator of the final term in $K_{a b}$ by $i \epsilon+E_{s}$, with the result that $K_{a b}$ then becomes a linear function of $E$. Before making this approximation, the kernel $K_{a b}$ has a non-trivial dependence on the stochasticity parameter $\sigma$. However, after making the Weisskopf-Wigner approximation, this $\sigma$ dependence completely cancels:

$$
\begin{aligned}
& \frac{1}{8} \sigma^{2}\left(V^{2}\right)_{s_{a} s_{b}}+\sum_{m \notin\left\{s_{a}\right\}} \frac{f_{m}^{2} V_{s_{a} m} V_{m s_{b}}}{-i E+i E_{s}+i\left(E_{m}-E_{s}\right) f_{m}} \\
\rightarrow & \frac{1}{8} \sigma^{2}\left(V^{2}\right)_{s_{a} s_{b}}+\sum_{m \notin\left\{s_{a}\right\}} \frac{f_{m}^{2} V_{s_{a} m} V_{m s_{b}}}{\epsilon+i\left(E_{m}-E_{s}\right) f_{m}} \\
= & \frac{1}{8} \sigma^{2}\left(V^{2}\right)_{s_{a} s_{b}}+\sum_{m \notin\left\{s_{a}\right\}} \frac{f_{m} V_{s_{a} m} V_{m s_{b}}}{\epsilon+i\left(E_{m}-E_{s}\right)} \\
= & \frac{1}{8} \sigma^{2}\left(V^{2}\right)_{s_{a} s_{b}}+\sum_{m \notin\left\{s_{a}\right\}} \frac{\left[1-(i / 8) \sigma^{2}\left(E_{m}-E_{s}\right)\right] V_{s_{a} m} V_{m s_{b}}}{\epsilon+i\left(E_{m}-E_{s}\right)} \\
= & \sum_{m \notin\left\{s_{a}\right\}} \frac{-i V_{s_{a} m} V_{m s_{b}}}{E_{m}-E_{s}-i \epsilon},
\end{aligned}
$$

where in the final step we have made use of the condition of Eq. (9a). Thus in the WeisskopfWigner approximation, the kernel $K_{a b}$ appearing in Eq. (17b) simplifies to

$$
\begin{aligned}
K_{a b} & =\left(-i E+i E_{s}\right) \delta_{a b}+\sum_{m \notin\left\{s_{a}\right\}} \frac{-i V_{s_{a} m} V_{m s_{b}}}{E_{m}-E_{s}-i \epsilon} \\
& =\left(-i E+i E_{s}\right) \delta_{a b}+i M_{a b}+\frac{1}{2} \Gamma_{a b}, \\
M_{a b} & =\sum_{m \notin\left\{s_{a}\right\}} P \frac{V_{s_{a} m} V_{m s_{b}}}{E_{s}-E_{m}}, \\
\Gamma_{a b} & =2 \pi \sum_{m \notin\left\{s_{a}\right\}} V_{s_{a} m} V_{m s_{b}} \delta\left(E_{m}-E_{s}\right),
\end{aligned}
$$


with $P$ in the definition of the "mass matrix" $M_{a b}$ the principal value. These are the same as the formulas for the kernel in the absence of the stochastic terms in the Schrödinger equation. Thus, in the Weisskopf-Wigner approximation, the solution for $E\left[C_{s_{a}}(t)\right]$ is unmodified by the stochastic effects, and hence the Lorentzian line profile and the decay rate of the state are unaffected by the $\sigma$ terms.

The solution for $E\left[C_{m}(t)\right]$ with $m \notin\left\{s_{a}\right\}$ does retain a dependence on the stochastic parameter. To study this, let us specialize to the case $D=1$ of a non-degenerate initial state. The expression in Eq. (18b) for the kernel now becomes the $1 \times 1$ matrix

$$
K(E)=-i E+i E_{s}+i M+\frac{1}{2} \Gamma,
$$

with $M$ and $\Gamma$ real numbers given by

$$
\begin{aligned}
M & =\sum_{m \neq s} P \frac{V_{s m} V_{m s}}{E_{s}-E_{m}}, \\
\Gamma & =2 \pi \sum_{m \neq s} V_{s m} V_{m s} \delta\left(E_{m}-E_{s}\right) .
\end{aligned}
$$

Thus, Eq. (17b) has the immediate solution

$$
g_{s}\left(-i E+i E_{s}\right)=K(E)^{-1},
$$

which when substituted into Eq. (17a) yields

$$
g_{m}\left(-i E+i E_{m}\right)=\left[-i E+i E_{m}+\frac{1}{8} \sigma^{2}\left(E_{m}-E_{s}\right)^{2}\right]^{-1} V_{m s} f_{m}\left(E-E_{s}-M+\frac{i}{2} \Gamma\right)^{-1} .
$$

Substituting these equations into the inversion formulas of Eqs. (17d), and doing elementary contour integrations, we find

$$
\begin{aligned}
E\left[C_{s}(t)\right] & =\exp \left(-i M t-\frac{1}{2} \Gamma t\right), \\
E\left[C_{m \neq s}(t)\right] & =\frac{V_{m s}}{E_{s}-E_{m}+M-\frac{i}{2} \Gamma}\left(\exp \left[i\left(E_{m}-E_{s}-M\right) t-\frac{1}{2} \Gamma t\right]-\exp \left[-\frac{1}{8} \sigma^{2}\left(E_{m}-E_{s}\right)^{2} t\right]\right) .
\end{aligned}
$$

¿From Eq. (11b) we thus get

$$
E\left[\left|C_{s}(t)\right|^{2}\right]=\exp (-\Gamma t)
$$

which identifies $\Gamma$ as the transition rate per unit time out of the initial state. Finally, substituting Eq. (20a) into Eq. (12b), simplifying to leading order in $V$, and integrating 
with respect to $t$, we get

$$
\begin{aligned}
E\left[\left|C_{m \neq s}(t)\right|^{2}\right]= & \frac{\left|V_{m s}\right|^{2}}{\left(E_{s}-E_{m}+M\right)^{2}+\frac{1}{4} \Gamma^{2}}(\exp (-\Gamma t)+1 \\
& \left.-2 \exp \left[-\frac{1}{8} \sigma^{2}\left(E_{m}-E_{s}\right)^{2} t-\frac{1}{2} \Gamma t\right] \cos \left[\left(E_{s}-E_{m}+M\right) t\right]\right) .
\end{aligned}
$$

This completes our solution for the expectations of the coefficients, and their squared magnitudes, in the case of a non-degenerate initial state. We see that after a time $t$ large compared with the lifetime $\Gamma^{-1}$, we obtain

$$
E\left[\left|C_{m \neq s}(\infty)\right|^{2}\right]=\frac{\left|V_{m s}\right|^{2}}{\left(E_{s}-E_{m}+M\right)^{2}+\frac{1}{4} \Gamma^{2}},
$$

exhibiting the standard Lorentzian profile with no dependence on the stochasticity parameter $\sigma$.

\section{SMALL TIME AND GOLDEN RULE APPROXIMATIONS}

Let us now study the behavior of Eq. (20g) for small and large values of the time $t$. Since within our approximations we have $\sigma^{2}\left(E_{m}-E_{s}\right)^{2} \simeq \sigma^{2}\left[\left(E_{s}-E_{m}+M\right)^{2}+\frac{1}{4} \Gamma^{2}\right]$, we can rewrite Eq. (200) as

$$
\begin{aligned}
E\left[\left|C_{m \neq s}(t)\right|^{2}\right]= & \frac{\left|V_{m s}\right|^{2}}{\left(E_{s}-E_{m}+M\right)^{2}+\frac{1}{4} \Gamma^{2}}(\exp (-\Gamma t)+1 \\
& \left.-2 \exp \left[-\frac{1}{8} \sigma^{2}\left(\left(E_{s}-E_{m}+M\right)^{2}+\frac{1}{4} \Gamma^{2}\right) t-\frac{1}{2} \Gamma t\right] \cos \left[\left(E_{s}-E_{m}+M\right) t\right]\right) .
\end{aligned}
$$

In the limit as $t \rightarrow 0$, we can develop the exponential and cosine functions in power series expansions, giving

$$
E\left[\left|C_{m \neq s}(t)\right|^{2}\right] \simeq\left|V_{m s}\right|^{2}\left(\frac{1}{4} \sigma^{2} t+t^{2}+O\left(t^{2} \sigma^{4}\left(E_{s}-E_{m}\right)^{2}\right)+O\left(t^{3}\right)\right) .
$$

Thus the leading small time behavior of the summed expected probability in the decay channels is

$$
\sum_{m \neq s}\left|V_{m s}\right|^{2} \frac{1}{4} \sigma^{2} t=\left(V^{2}\right)_{s s} \frac{1}{4} \sigma^{2} t
$$

where in evaluating the sum we have employed the condition of Eq. (9a). We shall verify this result by another method in Sec. [X], where we discuss its implications for the quantum Zeno effect, and in Sec. \ shall apply it to estimating bounds on $\sigma$. 
Let us next consider the large time behavior implied by Eq. (21a). Once $t$ is large enough so that $\left|\left(E_{m}-E_{s}\right) t\right|$ is large for all energies $E_{m}$ not infinitesimally close to $E_{s}$, we can evaluate the summed expected probability in the decay channels by making the "golden rule" approximation [12]. This approximation treats the factors multiplying $\left|V_{m s}\right|^{2}$ in Eq. (21a), which are sharply peaked around $E_{m}=E_{s}$, as if they were equal to a Dirac delta function of strength given by the integral of these factors over energy. We then have

$$
\begin{aligned}
\sum_{m \neq s} E\left[\left|C_{m \neq s}(t)\right|^{2}\right] & =\sum_{m \neq s} \frac{\left|V_{m s}\right|^{2}}{\left(E_{s}-E_{m}+M\right)^{2}+\frac{1}{4} \Gamma^{2}}(\exp (-\Gamma t)+1 \\
& \left.-2 \exp \left[-\frac{1}{8} \sigma^{2}\left(\left(E_{s}-E_{m}+M\right)^{2}+\frac{1}{4} \Gamma^{2}\right) t-\frac{1}{2} \Gamma t\right] \cos \left[\left(E_{s}-E_{m}+M\right) t\right]\right) \\
& \simeq \sum_{m \neq s}\left|V_{m s}\right|^{2} \delta\left(E_{m}-E_{s}\right) \\
& \times \int_{-\infty}^{\infty} d(\Delta E) \frac{\exp (-\Gamma t)+1-2 \exp \left[-\frac{1}{8} \sigma^{2}\left((\Delta E)^{2}+\frac{1}{4} \Gamma^{2}\right) t-\frac{1}{2} \Gamma t\right] \cos [(\Delta E) t]}{(\Delta E)^{2}+\frac{1}{4} \Gamma^{2}} \\
& =\sum_{m \neq s}\left|V_{m s}\right|^{2} \delta\left(E_{m}-E_{s}\right) t F\left[\sigma^{2} /(8 t), t\right] \\
& =\frac{\Gamma t}{2 \pi} F\left[\sigma^{2} /(8 t), t\right],
\end{aligned}
$$

with $\Gamma$ as given in Eq. (19b) and with the function $F[A, t]$ defined by

$$
F[A, t]=\int_{-\infty}^{\infty} d u \frac{\exp (-\Gamma t)+1-2 \exp \left[-A\left(u^{2}+\frac{1}{4} \Gamma^{2} t^{2}\right)-\frac{1}{2} \Gamma t\right] \cos u}{u^{2}+\frac{1}{4} \Gamma^{2} t^{2}} .
$$

To evaluate $F[A, t]$ we note that 13

$$
F[0, t]=\int_{-\infty}^{\infty} d u \frac{\exp (-\Gamma t)+1-2 \exp \left(-\frac{1}{2} \Gamma t\right) \cos u}{u^{2}+\frac{1}{4} \Gamma^{2} t^{2}}=\frac{2 \pi}{\Gamma t}[1-\exp (-\Gamma t)],
$$

and 13

$$
\begin{aligned}
\frac{\partial F[A, t]}{\partial A} & =\int_{-\infty}^{\infty} d u 2 \exp \left[-A\left(u^{2}+\frac{1}{4} \Gamma^{2} t^{2}\right)-\frac{1}{2} \Gamma t\right] \cos u \\
& =2 \pi^{1 / 2} \exp \left(-\frac{1}{2} \Gamma t-\frac{1}{4} A \Gamma^{2} t^{2}\right) A^{-1 / 2} \exp [-1 /(4 A)]
\end{aligned}
$$

Thus, integrating Eq. (23b) with respect to $A$ we get

$$
F[A, t]=\frac{2 \pi}{\Gamma t}[1-\exp (-\Gamma t)]+C[A, t],
$$

with the correction term $C[A, t]$ given by

$$
C[A, t]=4 \pi^{1 / 2} \exp \left(-\frac{1}{2} \Gamma t\right) \int_{0}^{A^{1 / 2}} d v \exp \left[-\frac{1}{4}\left(v^{2} \Gamma^{2} t^{2}+1 / v^{2}\right)\right] .
$$


Since the exponentials of negative arguments in Eq. (24a) are bounded by their maxima over the range of integration, we have

$$
|C[A, t]|<4 \pi^{1 / 2} A^{1 / 2} \exp [-1 /(4 A)]=2 \sigma\left(\frac{\pi}{2 t}\right)^{1 / 2} \exp \left(-2 t / \sigma^{2}\right) .
$$

So when $\Gamma t$ is of order unity, the contribution of the correction term $C[A, t]$ is of order $c \sigma \Gamma^{1 / 2} \exp \left[-2 /\left(\sigma^{2} \Gamma\right)\right] \sim c^{\prime} \sigma V \exp \left[-c^{\prime \prime} /(\sigma V)^{2}\right]$, with $c, c^{\prime}, c^{\prime \prime}$ constants, which is exponentially small and can be neglected in our approximation scheme. Thus we are justified in approximating

$$
F[A, t] \simeq F[0, t]=\frac{2 \pi}{\Gamma t}[1-\exp (-\Gamma t)],
$$

which when substituted back into Eq. (22a) gives

$$
\sum_{m \neq s} E\left[\left|C_{m \neq s}(t)\right|^{2}\right]=1-\exp (-\Gamma t)=1-\left|C_{s}(t)\right|^{2}
$$

verifying that the approximations used in our calculation are consistent with maintenance of the unitarity sum rule (the unit state vector normalization condition).

\section{SOLUTION TO THE STOCHASTIC EQUATION FOR $C_{m \neq s}$}

Since we see from Eqs. (20a) and (20c) that $E\left[\left|C_{m \neq s}(t)\right|^{2}\right.$ differs from $\left|E\left[C_{m \neq s}(t)\right]\right|^{2}$, the stochastic fluctuations in $C_{m \neq s}(t)$ are evidently playing a role. Let us now demonstrate this directly by solving the stochastic differential equation for $C_{m \neq s}(t)$. Specializing to the case of a non-degenerate initial state, approximating $C_{s}(t) \simeq E\left[C_{s}(t)\right]$, and using Eq. (20a) for $E\left[C_{s}(t)\right]$, Eq. (10d) becomes

$$
\begin{aligned}
d C_{m}(t) & =\left(\alpha_{m}^{(1)} d W_{t}+\alpha_{m}^{(2)} d t\right) C_{m}(t) \\
& +\exp \left[i\left(E_{m}-E_{s}-M\right) t-\frac{1}{2} \Gamma t\right]\left(\gamma_{m s}^{(1)} d W_{t}+\gamma_{m s}^{(2)} d t\right) \\
\alpha_{m}^{(1)} & =\frac{1}{2} \sigma\left(E_{m}-E_{s}\right), \quad \alpha_{m}^{(2)}=-\frac{1}{8} \sigma^{2}\left(E_{m}-E_{s}\right)^{2}=-\frac{1}{2}\left(\alpha_{m}^{(1)}\right)^{2} \\
\gamma_{m s}^{(1)} & =\frac{1}{2} \sigma V_{m s}, \quad \gamma_{m s}^{(2)} \simeq-i V_{m s} f_{m} .
\end{aligned}
$$

For general values of the coefficients $\alpha_{m}^{(1,2)}$ and $\gamma_{m s}^{(1,2)}$, Eq. 25a) can be integrated by using Eqs. (2 $2 \mathrm{a}-\mathrm{c}$ ) to find a stochastic integrating factor for the $C_{m}$ terms (see the Appendix), with the result

$$
\begin{aligned}
C_{m}(t) & =\exp \left[\alpha_{m}^{(1)} W_{t}-\left(\alpha_{m}^{(1)}\right)^{2} t\right] \int_{0}^{t} \exp \left[i\left(E_{m}-E_{s}-M\right) u-\frac{1}{2} \Gamma u-\alpha_{m}^{(1)} W_{u}+\left(\alpha_{m}^{(1)}\right)^{2} u\right] \\
& \times\left[\gamma_{m s}^{(1)} d W_{u}+\left(\gamma_{m s}^{(2)}-\alpha_{m}^{(1)} \gamma_{m s}^{(1)}\right) d u\right] .
\end{aligned}
$$


Using Eqs. (2a-c), it is easy to verify directly that Eq. (25b) solves Eq. 25a). If we now examine Eq. (25b) more closely, using the specific expressions for the coefficients $\alpha_{m}^{(1,2)}$ and $\gamma_{m s}^{(1,2)}$ given in Eq. (25a), we find that within the approximation of neglecting terms of relative order $\sigma V^{2}$, the integrand in Eq. (25b) is an exact stochastic differential. Thus the integration can be carried out explicitly (see the Appendix), with the result

$$
\begin{aligned}
C_{m \neq s}(t) & =\frac{V_{m s}}{E_{s}-E_{m}+M-\frac{i}{2} \Gamma} \\
& \times\left(\exp \left[i\left(E_{m}-E_{s}-M\right) t-\frac{1}{2} \Gamma t\right]-\exp \left[\frac{1}{2} \sigma\left(E_{m}-E_{s}\right) W_{t}-\frac{1}{4} \sigma^{2}\left(E_{m}-E_{s}\right)^{2} t\right]\right) .
\end{aligned}
$$

This expression can be easily verified, by use of Eqs. (2a-c), to be the solution to Eq. (25a) (up to a residual error of relative order $\sigma V^{2}$ ). Using Eq. (36) to take the expectation of Eq. (25d), we recover the result of Eq. (20a). From Eq. (25d) we find an explicit formula for $\left|C_{m \neq s}(t)\right|^{2}$,

$$
\begin{aligned}
\left|C_{m \neq s}(t)\right|^{2} & =\frac{\left|V_{m s}\right|^{2}}{\left(E_{s}-E_{m}+M\right)^{2}+\frac{1}{4} \Gamma^{2}} \\
& \times\left(\exp (-\Gamma t)+\exp \left[\sigma\left(E_{m}-E_{s}\right) W_{t}-\frac{1}{2} \sigma^{2}\left(E_{m}-E_{s}\right)^{2} t\right]\right. \\
& \left.-2 \exp \left[\frac{1}{2} \sigma\left(E_{m}-E_{s}\right) W_{t}-\frac{1}{4} \sigma^{2}\left(E_{m}-E_{s}\right)^{2} t-\frac{1}{2} \Gamma t\right] \cos \left(E_{m}-E_{s}-M\right) t\right) .
\end{aligned}
$$

Again using Eq. (30) to take the expectation of this formula, we recover the result of Eq. (200).

\section{STOCHASTIC SUPPRESSION OF THE QUANTUM ZENO EFFECT}

In Eqs. (21]b,c) we saw that the leading small time behavior of the summed expected probability in the decay channels is

$$
\left(V^{2}\right)_{s s} \frac{1}{4} \sigma^{2} t
$$

rather than the result $\left(V^{2}\right)_{s s} t^{2}$ that would hold for vanishing $\sigma$. As a result, $E\left[\left|C_{s}(t)\right|^{2}\right]-1$ vanishes linearly in $t$ for nonzero $\sigma$, rather than quadratically in $t$ as for the unmodified Schrödinger equation. Since the quadratic vanishing of $\left|C_{s}(t)\right|^{2}-1$ in standard quantum mechanics is the origin of the quantum Zeno effect [14], we conclude that in the energy driven stochastic Schrödinger equation, the quantum Zeno effect is suppressed. 
Let us verify this directly from the stochastic differential equation of Eq. (10), in analogy with the direct calculation [15] of $\left|C_{s}(t)\right|^{2}-1$ for small times for the ordinary Schrödinger equation. Applying the Itô rule of Eq. (2a), we have

$\left.d|\langle s(0) \mid s(t)\rangle|^{2}\right|_{t=0}=\left.\langle s(0)|d| s(t)\rangle\right|_{t=0}+\left.\langle s(0)|d| s(t)\rangle^{*}\right|_{t=0}+\left.\left.\langle s(0)|d| s(t)\rangle\right|_{t=0}\langle s(0)|d| s(t)\rangle^{*}\right|_{t=0} \quad$.

From Eq. (10) we have

$d|s(t)\rangle=-i H|s(t)\rangle d t-\frac{1}{8} \sigma^{2}(H-\langle s(t)|H| s(t)\rangle)^{2}|s(t)\rangle d t+\frac{1}{2} \sigma(H-\langle s(t)|H| s(t)\rangle)|s(t)\rangle d W_{t}$,

and so setting $t=0$ and projecting on $\langle s(0)|$ gives

$$
\left.\langle s(0)|d| s(t)\rangle\right|_{t=0}=-i\langle H\rangle_{s} d t-\frac{1}{8} \sigma^{2}\left\langle\left(H-\langle H\rangle_{s}\right)^{2}\right\rangle_{s} d t
$$

with $\left\langle H^{n}\right\rangle_{s}=\left\langle s(0)\left|H^{n}\right| s(0)\right\rangle$. Substituting Eq. (27d) into Eq. (27a), we thus get the first term in the small $t$ expansion of $|\langle s(0) \mid s(t)\rangle|^{2}-1$,

$$
|\langle s(0) \mid s(t)\rangle|^{2}=1-\frac{1}{4} \sigma^{2}\left\langle\left(H-\langle H\rangle_{s}\right)^{2}\right\rangle_{s} t+O\left(t^{2}\right) .
$$

This equation gives a general formula for the stochastic suppression of the quantum Zeno effect, independent of any assumptions about the potential. When the general form [15] of the order $t^{2}$ term coming from the standard Schrödinger evolution is included, Eq. (28a) becomes

$$
|\langle s(0) \mid s(t)\rangle|^{2}=1-\left\langle\left(H-\langle H\rangle_{s}\right)^{2}\right\rangle_{s}\left(\frac{1}{4} \sigma^{2} t+t^{2}\right)+O\left(\sigma^{4} t^{2}\right)+O\left(t^{3}\right) \quad ;
$$

in other words, the first two terms in the small $t$ expansion are governed to leading order in $\sigma$ by the initial state energy variance. When the potential is assumed to obey Eq. (9a), we have

$$
\begin{gathered}
\langle H\rangle_{s}=E_{s}+V_{s s}=E_{s} \\
\left\langle H^{2}\right\rangle_{s}=E_{s}^{2}+2 E_{s} V_{s s}+\left(V^{2}\right)_{s s}, \\
\left\langle\left(H-\langle H\rangle_{s}\right)^{2}\right\rangle_{s}=\left\langle H^{2}\right\rangle_{s}-\langle H\rangle_{s}^{2}=\left(V^{2}\right)_{s s},
\end{gathered}
$$

and so Eq. (28b) becomes

$$
|\langle s(0) \mid s(t)\rangle|^{2}=1-\left(V^{2}\right)_{s s}\left(\frac{1}{4} \sigma^{2} t+t^{2}\right)+O\left(\sigma^{4} t^{2}\right)+O\left(t^{3}\right),
$$

in agreement with the result of Eqs. (21a,b) and the unitarity sum rule. 


\section{DISCUSSION AND ESTIMATES OF BOUNDS ON $\sigma$}

We have seen that to leading order in the perturbing potential, the stochastic terms governed by $\sigma$ do not affect either the Lorentzian line profile or the transition rate per unit time as evaluated in the Weisskopf-Wigner approximation, but only produce a change in the short time transient behavior of the transition probabilities from the initial state. This is a direct result of the fact that the energy-driven stochastic Schrödinger equation is energy conserving. On dimensional grounds, the transition rate per unit time $\Gamma$ could contain, in addition to the usual terms of the form $\delta\left(E_{s}-E_{m}\right)\left|V_{m s}\right|^{2}$, a term of the form $\sigma^{2}\left(V^{2}\right)_{s s}$. However, this additional term is not energy conserving, and as a result we have seen that its coefficient precisely cancels to zero in the Weisskopf-Wigner approximation.

Because the transition rate per unit time and Lorentzian line shape are unaffected by $\sigma$, bounds on $\sigma$ from particle decays result only from experiments in which a metastable system is monitored as function of time from a known time (or vertex location) of formation. According to Eqs. (28a-d), for small times the effective transition rate per unit time is

$$
\Gamma_{R}=\frac{1}{4} \sigma^{2}(\Delta E)^{2}=\frac{1}{4} \sigma^{2}\left(V^{2}\right)_{s s}
$$

with $(\Delta E)^{2}=\left\langle\left(H-\langle H\rangle_{s}\right)^{2}\right\rangle_{s}$ the initial state energy variance. This can be interpreted as an early time decay rate coming from spontaneous reduction induced by the stochastic fluctuation terms, in agreement with the estimate $\Gamma_{R} \sim \sigma^{2}(\Delta E)^{2}$ used in earlier discussions [4, 6]. In order for the rate of Eq. (29a) to not lead to pronounced early time deviations from the observed decay rate $\Gamma$, we must have

$$
\Gamma_{R}<\Gamma,
$$

which writing $\sigma^{2}=M_{\sigma}^{-1}$ implies the bound

$$
M_{\sigma}>\frac{\left(V^{2}\right)_{s s}}{4 \Gamma}=\frac{\sum_{m \neq s}\left|V_{s m}\right|^{2}}{8 \pi \sum_{m \neq s}\left|V_{s m}\right|^{2} \delta\left(E_{m}-E_{s}\right)} \equiv \frac{E_{D}}{8 \pi}
$$

with $E_{D}$ defining an energy characteristic of the decay process. In a particle physics context, a first guess would be to estimate $E_{D}$ as being of order the mass of the decaying particle. The most massive decays for which $\Gamma$ has been measured by tracking a metastable system

from the point of formation appear to be $\pi^{0} \rightarrow \gamma \gamma$ decay, with an initial mass order 140 $\mathrm{MeV}$, and charmed meson decays, with an initial mass of around $2 \mathrm{GeV}$. Estimating $E_{D}$ in 
Eq. (30) as the decaying particle mass, these give respective bounds on $M_{\sigma}$ of order $6 \mathrm{MeV}$ and $80 \mathrm{MeV}$, respectively. If $M_{\sigma}$ were significantly larger than these bounds, one would have observed anomalous accumulations of decay events close to the production vertex, as a result of decays induced by spontaneous reduction. For comparison, the observation of coherent superpositions of energy eigenstates in the neutrino, $K$-meson and $B$-meson systems gives bounds [16], respectively, of $M_{\sigma}>10^{-20} \mathrm{GeV}, M_{\sigma}>2 \times 10^{-15} \mathrm{GeV}$, and $M_{\sigma}>2 \times 10^{-13} \mathrm{GeV}$.

Thus the charmed meson decay bound on $M_{\sigma}$ represents a significant improvement over the coherent oscillation bounds. However, it is still smaller than the Planck mass, which is very likely the expected value of $M_{\sigma}$, by a factor of $10^{20}$ ! We conclude that the theory of decaying states in the energy-driven stochastic Schrödinger equation places only very weak empirical bounds on the magnitude of the stochasticity parameter $\sigma$.

We leave for future study two issues that can be addressed within the general framework established here. The first is an analysis of the nature of the transition between the shorttime regime with decay rate $\Gamma_{R}$, and the exponential decay regime with decay rate $\Gamma$. This is governed by the solution of Eqs. (17a,b) before making the Weisskopf-Wigner approximation of replacing $E$ in the denominator of the final term in $K_{a b}$ by $E_{s}$. The second is an analysis of the magnitude of the energy $E_{D}$ defined by Eq. (30), for various dynamical models of the decay process, as reflected in the energy spectrum of the unperturbed states $|m\rangle$ and in the magnitudes of the decay-inducing matrix elements $V_{s m}$.

\section{ACKNOWLEDGMENTS}

This work was supported in part by the Department of Energy under Grant \#DE-FG0290ER40542. I wish to acknowledge the hospitality of the Aspen Center for Physics, where most of the calculations reported here were done. I also wish to thank Angelo Bassi, Dorje Brody, Todd Brun, and especially Lane Hughston, for informative discussions about the properties of the Itô calculus, Todd Brun for a helpful discussion about extracting bounds from decay observations, Larry Horwitz for a discussion several years ago about virtues of the Laplace transform, and Edward Witten for raising the issue of empirical bounds on the stochasticity parameter. 


\section{ADDED NOTE}

Lajos Diósi [17] has pointed out an elegant stochastic-theoretic technique that allows the main physical results of this paper to be derived in a few lines, starting from the standard quantum mechanical results that hold when the stochasticity parameter $\sigma$ is zero. Diósi makes three principal observations. The first is that the quantities of direct physical interest, as pointed out in Sec. $\mathrm{V}$, are the expectations $E\left[\left|C_{m}(t)\right|^{2}\right]$ of the squared magnitudes of the perturbation coefficients. Since according to Eq. (6b) we have

$$
\left|C_{m}(t)\right|^{2}=|\langle m \mid \psi(t)\rangle|^{2}=\langle m \mid \psi(t)\rangle\langle\psi(t) \mid m\rangle=\langle m|\rho(t)| m\rangle
$$

with $\rho(t)$ the density matrix

$$
\rho(t)=|\psi(t)\rangle\langle\psi(t)|
$$

to calculate $E\left[\left|C_{m}(t)\right|^{2}\right.$ it suffices to know $E[\rho(t)]$, in other words

$$
E\left[\left|C_{m}(t)\right|^{2}\right]=\langle m|E[\rho(t)]| m\rangle=\operatorname{Tr}(|m\rangle\langle m|) E[\rho(t)] \quad .
$$

Since the dynamics of $E[\rho(t)]$ is governed by the Lindblad-type equation of Eq. (5), to calculate the physically relevant expectations it thus suffices to solve the dynamical problem specified by Eq. (5), supplemented by the initial condition

$$
E[\rho(0)]=\rho(0)=\left|s_{A}\right\rangle\left\langle s_{A}\right|
$$

Diósi's second observation is that the dynamical problem specified by Eq. (5), with the initial condition of Eq. (31d), can be compactly solved by a simple stochastic trick. The trick uses the fact [18 that there is a second stochastic Schrödinger equation, simpler in structure than that of Eq. (1a), which also leads to Eq. (5) as the equation for the evolution of the stochastic expectation of its density matrix. To see this, consider the stochastic Schrödinger equation

$$
d|\psi\rangle=-i H|\psi\rangle d t-\frac{1}{8} \sigma^{2} H^{2}|\psi\rangle d t+\frac{1}{2} i \sigma H|\psi\rangle d W_{t}
$$

This equation differs from that of Eq. (1a) in having an imaginary noise term, with operator coefficient $H$, instead of a real noise term with operator coefficient $H-\langle H\rangle$. A simple

calculation, using the Itô calculus rules of Eq. (11b), shows that Eq. (32a) also leads to preservation of the norm of the state $|\psi\rangle$, and leads to the density matrix evolution equation

$$
d \rho=i[\rho, H] d t-\frac{1}{8} \sigma^{2}[H,[H, \rho]] d t+\frac{1}{2} i \sigma[H, \rho] d W_{t}
$$


which has the stochastic expectation

$$
d E[\rho]=i[E[\rho], H] d t-\frac{1}{8} \sigma^{2}[H,[H, E[\rho]]] d t \quad,
$$

which is identical to Eq. (5). Hence the imaginary noise equation of Eq. (32a) will lead to the same results for the physical quantities $E\left[\left|C_{m}(t)\right|^{2}\right]$ as the real noise equation of Eq. (1a), even though the stochastic details of the two processes differ!

Diósi's third observation is the fact that Eq. (32a) can be immediately formally integrated to give

$$
|\psi(t)\rangle=\exp \left[-i H\left(t-\frac{1}{2} \sigma W_{t}\right)\right] \mid \psi(0\rangle
$$

as can be readily ascertained by use of Eq. (2d) with the choice

$$
\alpha=\frac{1}{2} i \sigma H
$$

Combining this observation with the first two, then leads to a very simple rule for calculating the stochastic modifications of decay processes governed by Eq. (1a). Let $E\left[\left|C_{m}^{\sigma}(t)\right|^{2}\right]$ be the quantities of physical interest, viewed as functions of $\sigma$ as well as of $t$, so that $E\left[\left|C_{m}^{0}(t)\right|^{2}\right]=$ $\left|C_{m}^{0}(t)\right|^{2}$ are their values as calculated from the standard Schrödinger evolution with no stochasticity. Then Eqs. (31a) through (33b) imply the simple relation

$$
E\left[\left|C_{m}^{\sigma}(t)\right|^{2}\right]=E\left[\left|C_{m}^{0}\left(t-\frac{1}{2} \sigma W_{t}\right)\right|^{2}\right],
$$

between the probabilities calculated in the standard Schrödinger analysis, and the stochastic expectations of the probabilities as calculated from Eq. (1a $)$. The recipe is simply this: take the known expressions for the probabilities calculated in standard quantum mechanics, replace $t$ by $t-\frac{1}{2} \sigma W_{t}$, and take the stochastic expectation. The needed stochastic expectations of powers of $W_{t}$ can all be read off from the expansion of Eq. (3c) in powers of $\alpha$,

$$
E\left[W_{t}\right]=0, \quad E\left[W_{t}^{2}\right]=t, E\left[W_{t}^{3}\right]=0, \quad E\left[W_{t}^{4}\right]=3 t^{2}, \ldots
$$

Let us now apply Diósi's observations to rederive the principal results found above for the stochastic analog of the Weisskopf-Wigner analysis. First, let us consider the short time behavior of the survival probability given in Eq. (28b). The standard answer when $\sigma=0$, which gives the quantum Zeno effect, is

$$
|\langle s(0) \mid s(t)\rangle|^{2}=1-\left\langle\left(H-\langle H\rangle_{s}\right)^{2}\right\rangle_{s} t^{2}+O\left(t^{3}\right)
$$


Following the recipe, we have

$$
E\left[\left(t-\frac{1}{2} \sigma W_{t}\right)^{2}\right]=E\left[t^{2}-t \sigma W_{t}+\frac{1}{4} \sigma^{2} W_{t}^{2}\right]=t^{2}+\frac{1}{4} \sigma^{2} t .
$$

On substitution into Eq. (35a) this gives for general $\sigma$

$$
|\langle s(0) \mid s(t)\rangle|^{2}=1-\left\langle\left(H-\langle H\rangle_{s}\right)^{2}\right\rangle_{s}\left(t^{2}+\frac{1}{4} \sigma^{2} t\right)+\ldots,
$$

in agreement with the result for the stochastic modification of the quantum Zeno effect given in Eq. (28b).

Next let us apply the recipe to the formula for the initial state survival probability obtained using the Weisskopf-Wigner approximation, which is valid for times $t$ that are not too small (and also not too large). The standard analysis gives

$$
\left|C_{s}^{0}(t)\right|^{2}=\exp (-\Gamma t)
$$

with $\Gamma$ the Golden Rule decay rate of Eq. (19b). Replacing $t$ by $t-\frac{1}{2} \sigma W_{t}$ and using Eq. (3G) to take the stochastic expectation, we get as the exact formula for the stochastic modification of the Weisskopf-Wigner approximation

$$
E\left[\left|C_{s}^{\sigma}(t)\right|^{2}\right]=\exp \left[-\Gamma\left(1-\frac{1}{8} \sigma^{2} \Gamma\right) t\right],
$$

which reduces, when the correction term of relative order $\sigma^{2} \Gamma$ is neglected, to the answer found in Eq. (20b). Since $\sigma^{2} \Gamma \sim O\left(\sigma^{2} V^{2}\right)$, we see that the calculation of Secs. $4-6$ above did not succeed in keeping all terms of order $\sigma^{2} V^{2}$, and in fact there is a small stochastic correction to the decay rate, with the corrected decay rate given by

$$
\Gamma^{\sigma}=\Gamma\left(1-\frac{1}{8} \sigma^{2} \Gamma\right)
$$

However, writing $\sigma^{2}=M_{\sigma}^{-1}$ as in Sec. Х, as long as $M_{\sigma}>E_{s}$ this correction is not significant within the Weisskopf-Wigner approximation scheme, which treats the line width $\Gamma$ as a small quantity relative to $E_{s}$.

Finally, let us apply the recipe to the formula giving the probability for a transition to the state $|m\rangle$. The standard Weisskopf-Wigner approximation result for this is given by Eq. (20c) with $\sigma=0$,

$$
\begin{aligned}
\left|C_{m \neq s}^{0}(t)\right|^{2}= & \frac{\left|V_{m s}\right|^{2}}{\left(E_{s}-E_{m}+M\right)^{2}+\frac{1}{4} \Gamma^{2}}(\exp (-\Gamma t)+1 \\
& \left.-2 \exp \left[-\frac{1}{2} \Gamma t\right] \cos \left[\left(E_{s}-E_{m}+M\right) t\right]\right) .
\end{aligned}
$$


Applying the recipe, and again using Eq. (3G) to evaluate the needed expectations, we get the exact stochastic extension of Eq. (37a),

$$
\begin{aligned}
E\left[\left|C_{m \neq s}^{\sigma}(t)\right|^{2}\right]= & \frac{\left|V_{m s}\right|^{2}}{\left(E_{s}-E_{m}+M\right)^{2}+\frac{1}{4} \Gamma^{2}}\left(\exp \left[-\Gamma\left(1-\frac{1}{8} \sigma^{2} \Gamma\right) t\right]+1\right. \\
& -2 \exp \left[-\frac{1}{2} \Gamma\left(1-\frac{1}{16} \sigma^{2} \Gamma\right) t-\frac{1}{8} \sigma^{2}\left(E_{s}-E_{m}+M\right)^{2} t\right] \\
\times & \left.\cos \left[\left(E_{s}-E_{m}+M\right)\left(1-\frac{1}{8} \sigma^{2} \Gamma\right) t\right]\right) .
\end{aligned}
$$

Again, when simplified to leading order in $V$, this gives the result of Eq. (200) above. However, even before dropping nonleading terms in $V$, we see that Eq. (37b) implies the Lorentzian formula of Eq. (20d) in the large time limit.

From the above exposition, we see that Diósi's observations not only greatly simplify the calculation of the physically relevant quantities, but also give results that are completely independent of the assumption of Eq. (9a) that was used to linearize the stochastic equation. (This is something that one might have already suspected from the fact that Eq. (28b) is more general than Eq. (28d).) Thus, the only approximations that are needed to get stochastic results are those that are used in the standard, non-stochastic quantum mechanical analysis. Moreover, the "miraculous" cancellation of the $\sigma^{2}$ terms in the Weisskopf-Wigner approximation to the mass and decay matrices, exhibited above in Eq. (18a), is given a deeper explanation. There is an extensive literature [19] discussing the decay problem without making the Weisskopf-Wigner approximation (i.e., without replacing $E$ by $E_{s}$ in the order $V^{2}$ terms of the Laplace transform kernel $K_{a b}$ ), and these discussions can all be converted to results for $E\left[\left|C_{m}^{\sigma}(t)\right|^{2}\right]$ in the stochastic case, by using the recipe of replacing $t$ by $t-\frac{1}{2} \sigma W_{t}$ in the corresponding formula for $\left|C_{m}^{0}(t)\right|^{2}$ and taking a stochastic average over $W_{t}$.

The relation of Eq. (34a) between stochastic and standard quantum mechanical probabilities can be applied to other problems as well. For example, the density matrix of a two-level system can be represented in the form

$$
\rho=\frac{1}{2}(1-\vec{R} \cdot \vec{\tau})
$$

with $\vec{\tau}=\left(\tau_{1}, \tau_{2}, \tau_{3}\right)$ the standard Pauli matrices, and with $\vec{R}=\left(R_{1}, R_{2}, R_{3}\right)$ a vector summarizing the structure of the traceless part of the density matrix. The standard, $\sigma=0$ Schrödinger equation describing Rabi oscillations of the two-level system under the influence of an applied field oscillating at the frequency of the level separation (in co-rotating 
coordinates, neglecting the counter-rotating field component) gives for the equation of motion [20] of the vector $\left.\vec{R}\right|_{\sigma=0} \equiv \vec{R}^{0}$,

$$
\frac{d \vec{R}^{0}}{d t}=\vec{\omega} \times \vec{R}^{0}
$$

with $|\vec{\omega}|=\Omega$ the angular frequency of precession of $\vec{R}^{0}$. The probabilities for finding the system in the upper and lower levels are given, as a function of time, by

$$
P_{ \pm}^{0}(t)=\frac{1}{2}\left[1 \pm R_{3}^{0}(t)\right]
$$

Since the general solution of Eq. (38b) has the form

$$
\vec{R}^{0}(t)=\vec{V}_{1} \cos \Omega t+\vec{V}_{2} \sin \Omega t
$$

with $\vec{V}_{1,2}$ fixed vectors that depend on the initial state and the structure of the Hamiltonian, and since

$$
\begin{aligned}
& E\left[\cos \Omega\left(t-\frac{1}{2} \sigma W_{t}\right)\right]=\exp \left(-\frac{1}{8} \Omega^{2} \sigma^{2} t\right) \cos \Omega t \\
& E\left[\sin \Omega\left(t-\frac{1}{2} \sigma W_{t}\right)\right]=\exp \left(-\frac{1}{8} \Omega^{2} \sigma^{2} t\right) \sin \Omega t
\end{aligned}
$$

we have under the stochastic evolution of Eq. (1a)

$$
E\left[\vec{R}^{\sigma}(t)\right]=\exp \left(-\frac{1}{8} \Omega^{2} \sigma^{2} t\right) \vec{R}^{0}(t)
$$

By Eq. (380), this gives for the expected probabilities when the system evolves under the stochastic Schrödinger equation,

$$
\frac{1}{2}-E\left[P_{ \pm}^{\sigma}(t)\right]=\exp \left(-\frac{1}{8} \Omega^{2} \sigma^{2} t\right)\left[\frac{1}{2}-P_{ \pm}^{0}(t)\right]
$$

This can be applied, for example, to the quantum Zeno effect experiment of Itano et. al. [21], who carry out a proposal of Cook 22] to make repeated measurements of a two-level system while the vector $\vec{R}$ is precessing for a time interval $t=\pi / \Omega$, for which the exponential damping factor in Eq. (39g) becomes $\exp \left(-\frac{1}{8} \pi \Omega \sigma^{2}\right)$. Corresponding to the experimental value $\Omega=320.7 \mathrm{MHz}$ and the fact that probabilities were observed to an accuracy of about .02 in this experiment, and were found to agree with the standard Schrödinger theory, we get a bound on $M_{\sigma}=1 / \sigma^{2}$ of $M_{\sigma}>2 \times 10^{-15} \mathrm{GeV}$, comparable to that obtained from oscillations in the $K$-meson system. 


\section{APPENDIX}

We give here the details of the integration of the stochastic differential equation that appears in Sec. VIII. Consider the linear stochastic differential equation

$$
d C_{t}=\left(A_{t} d W_{t}+B_{t} d t\right) C_{t}+P_{t} d W_{t}+Q_{t} d t
$$

which is to be solved for the unknown stochastic function $C_{t}$ given the known functions $A_{t}$, $B_{t}, P_{t}$, and $Q_{t}$. Although we shall proceed as if these known functions were deterministic, in fact all our manipulations and the final solution are unchanged [23] if the input functions are themselves stochastic. To solve Eq. (A.1), we transpose the $C_{t}$ term on the right to the left and multiply by a factor $F_{t}$, which is to be determined, giving

$$
F_{t}\left[d C_{t}-\left(A_{t} d W_{t}+B_{t} d t\right) C_{t}\right]=F_{t}\left[P_{t} d W_{t}+Q_{t} d t\right]
$$

We now look for an $F_{t}$ which makes the left hand side of Eq. (A.2) a total differential, up to terms independent of $C_{t}$ that are of the same form as the terms on the right hand side. Making the Ansatz

$$
F_{t}=\exp \left[\int_{0}^{t}\left(\alpha_{u} d W_{u}+\beta_{u} d u\right)\right],
$$

we find by use of Eqs. (2a) and (20) of the text that

$$
d\left(F_{t} C_{t}\right)=F_{t}\left[d C_{t}+\alpha_{t} d W_{t} C_{t}+\left(\beta_{t}+\frac{1}{2} \alpha_{t}^{2}\right) d t C_{t}+\alpha_{t} d W_{t} d C_{t}\right],
$$

which on substituting Eq. (A.1) for the final $d C_{t}$ on the right, and using Eq. (10) of the text, gives

$$
d\left(F_{t} C_{t}\right)=F_{t}\left[d C_{t}+\alpha_{t} d W_{t} C_{t}+\left(\beta_{t}+\frac{1}{2} \alpha_{t}^{2}+\alpha_{t} A_{t}\right) d t C_{t}+\alpha_{t} P_{t} d t\right]
$$

Hence if we choose

$$
\alpha_{t}=-A_{t}, \quad \beta_{t}=-B_{t}+\frac{1}{2} A_{t}^{2},
$$

then Eq. (A.5a) takes the form

$$
d\left(F_{t} C_{t}\right)=F_{t}\left[d C_{t}-\left(A_{t} d W_{t}+B_{t} d t\right) C_{t}-A_{t} P_{t} d t\right]
$$

which by use of Eq. (A.2) becomes

$$
d\left(F_{t} C_{t}\right)=F_{t}\left[P_{t} d W_{t}+\left(Q_{t}-A_{t} P_{t}\right) d t\right] .
$$


The dependence on the unknown function $C_{t}$ is now entirely in the form of an exact differential, and so Eq. (A.7) can be immediately integrated to give

$$
\begin{aligned}
C_{t} & =F_{t}^{-1}\left[C_{0}+\int_{0}^{t} d u F_{u}\left(P_{u} d W_{u}+\left(Q_{u}-A_{u} P_{u}\right) d u\right)\right] \\
& =\exp \left[\int_{0}^{t}\left(A_{u} d W_{u}+\left(B_{u}-\frac{1}{2} A_{u}^{2}\right) d u\right)\right] \\
& \times\left(C_{0}+\int_{0}^{t} d u \exp \left[-\int_{0}^{u}\left(A_{v} d W_{v}+\left(B_{v}-\frac{1}{2} A_{v}^{2}\right) d v\right)\right]\left[P_{u} d W_{u}+\left(Q_{u}-A_{u} P_{u}\right) d u\right]\right),
\end{aligned}
$$

which is the general solution of Eq. (A.1).

In Sec. VII], we need only the case of Eq. (A.1) in which $A_{t}=A, B_{t}=B, P_{t}=P f_{t}$, and $Q_{t}=Q f_{t}$, with $A, B, P, Q$ constants and with $f_{t}$ of the form $f_{t}=\exp (K t)$, and so the solution of Eq. (A.8) then becomes

$$
\begin{aligned}
C_{t} & =\exp \left(A W_{t}+\left(B-\frac{1}{2} A^{2}\right) t\right) \\
& \times\left(C_{0}+\int_{0}^{t} d u \exp \left(-A W_{u}+\left(K-B+\frac{1}{2} A^{2}\right) u\right)\left[P d W_{u}+(Q-A P) d u\right]\right) .
\end{aligned}
$$

Using the identity (proved by the same methods used to find the integrating factor $F_{t}$ ),

$$
\begin{aligned}
& \exp \left(\alpha W_{u}+(\beta+K) u\right)\left[P d W_{u}+(Q+\alpha P) d u\right] \\
= & \frac{P}{\alpha} d \exp \left(\alpha W_{u}+(\beta+K) u\right)-\frac{P}{\alpha}\left(\beta+K-\frac{Q}{P} \alpha-\frac{1}{2} \alpha^{2}\right) \exp \left(\alpha W_{u}+(\beta+K) u\right) d u
\end{aligned}
$$

and taking $\alpha=-A$ and $\beta=-B+A^{2} / 2$, the $P d W_{u}$ term in Eq. (A.9) can be eliminated. This gives an alternate form for the solution $C_{t}$,

$$
\begin{aligned}
C_{t} & =\exp \left(A W_{t}+\left(B-\frac{1}{2} A^{2}\right) t\right) \\
& \times\left(C_{0}-\frac{P}{A}\left[\exp \left(-A W_{t}+\left(K-B+\frac{1}{2} A^{2}\right) t\right)-1\right]\right. \\
& \left.+\left[\frac{P}{A}(K-B)+Q\right] \int_{0}^{t} d u \exp \left(-A W_{u}+\left(K-B+\frac{1}{2} A^{2}\right) u\right)\right) .
\end{aligned}
$$

Taking

$$
\begin{aligned}
A & =\alpha_{m}^{(1)}=\frac{1}{2} \sigma\left(E_{m}-E_{s}\right), \\
B & =-\frac{1}{2}\left(\alpha_{m}^{(1)}\right)^{2}=-\frac{1}{8} \sigma^{2}\left(E_{m}-E_{s}\right)^{2}, \\
P & =\frac{1}{2} \sigma V_{m s}, \\
Q & =-i V_{m s} f_{m}, \quad f_{m}=1-\frac{i}{8} \sigma^{2}\left(E_{m}-E_{s}\right), \\
K & =i\left(E_{m}-E_{s}-M\right)-\frac{1}{2} \Gamma,
\end{aligned}
$$


in Eqs. (A.9) and (A.11) gives the results quoted respectively in Eqs. (25b) and (25c) of the text.

[1] J. Ellis, J. S. Hagelin, D. V. Nanopoulos, and M. Srednicki, Nucl. Phys. B 241, 381 (1984); J. Ellis, N. E. Mavromatos, and D. V. Nanopoulos, in "Proceedings of the 31st International School of Subnuclear Physics, Erice, Italy, 1993”, Subnuclear Series Vol. 31, A. Zichichi, ed. (World Scientific, Singapore, 1995); J. Ellis, N. E. Mavromatos, and D. V. Nanopoulos, Chaos Solitons Fractals 10, 345 (1999).

[2] R. Penrose, "On Gravity's Role in Quantum State Reduction", in C. Callender and N. Huggett, eds., "Physics Meets Philosophy at the Planck Scale: Contemporary Theories in Quantum Gravity", pp. 290-304 (Cambridge University Press, Cambridge, 2001).

[3] P. Pearle, Phys. Rev. D 13, 857 (1976); P. Pearle, Int. Journ. Theor. Phys. 18, 489 (1979); P. Pearle, Phys. Rev. D 29, 235 (1984); P. Pearle, Phys. Rev. A 39, 2277 (1989); G. C. Ghirardi, A. Rimini, and T. Weber, Phys. Rev. D 34, 470 (1986); G. C. Ghirardi, P. Pearle, and A. Rimini, Phys. Rev. A 42, 78 (1990); L. Diósi, Phys. Lett. A 129, 419 (1988). For recent reviews, see A. Bassi and G. C. Ghirardi, "Dynamical Reduction Models", Physics Reports (in press); P. Pearle, "Collapse Models", in H.-P. Breuer and F. Pettrucione, eds., "Open Systems and Measurements in Relativistic Quantum Field Theory" (Lecture Notes in Physics 526) (Springer, Berlin, 1999).

[4] N. Gisin, Phys. Rev. Lett. 52, 1657 (1984); N. Gisin, Helv. Phys. Acta 62, 363 (1989); I. Percival, Proc. Roy. Soc. London A 447, 189 (1994); L. P. Hughston, Proc. Roy. Soc. Lond. A 452, 953 (1996); S. L. Adler and L. P. Horwitz, J. Math. Phys. 41, 2485 (2000).

[5] I. Bialynicki-Birula and J. Mycielski, Ann. Phys. 100, 62 (1976); S. Weinberg, Nucl. Phys. B 6 (Proc. Suppl.),67 (1989); S. Weinberg, Phys. Rev. Lett. 62, 485 (1989); S. Weinberg, Ann. Phys. 194, 336 (1989); J. Polchinski, Phys. Rev. Lett. 66, 397 (1991); N. Gisin, J. Phys. A: Math. Gen. 28, 7375 (1995).

[6] S. L. Adler, D. C. Brody, T. A. Brun, and L. P. Hughston, J. Phys. A: Math. Gen. 34, 8795 (2001); S. L. Adler, J. Phys. A: Math. Gen. 34, 8795 (2001).

[7] E. Lisi, A. Marrone, and D. Montanino, Phys. Rev. Lett. 85, 1166 (2000); S. L. Adler, Phys. Rev. D 62, 117901 (2000); see also Ref. [16]. 
[8] V. F. Weisskopf and E. P. Wigner, Z. Phys. 63, 54 (1930); E. Merzbacher, "Quantum Mechanics", 2nd. ed., Chapt. 18 (John Wiley, New York, 1970).

[9] For a pedagogical introduction to the Itô calculus, see C. W. Gardiner, "Handbook of Stochastic Methods", Chapt. 4 (Springer-Verlag, Berlin, 1990).

[10] G. Lindblad, Commun. Math. Phys. 48, 119 (1976); V. Gorini, A. Kossakowski, and E. C. G. Sudarshan, J. Math. Phys. 17, 821 (1976).

[11] E. Merzbacher, Ref. [8].

[12] E. Merzbacher, Ref. [8], pp. 479-480.

[13] I. S. Gradshteyn and and I. M. Ryzhik, "Table of Integrals, Series and Products" (Academic Press, New York, 1965). In Eq. (23a) we use p. 406, 3.723 no. 2, and in Eq. (23b) we use p. 480, 3.896 no. 4 .

[14] B. Misra and E. C. G. Sudarshan, J. Math. Phys. 18, 756 (1977). For a recent discussion and further references, see P. Facchi and S. Pascazio, "Unstable Systems and Quantum Zeno Phenomena in Quantum Field Theory," quant-ph/0202127, and E. Joos, "Decoherence Through Interaction with the Environment," in D. Giulini, E. Joos, C. Kiefer, J. Kupsch, J.O. Stamatescu, and H.D. Zeh, "Decoherence and the Appearance of a Classical World in Quantum Theory," Sec. 3.3.1 (Springer-Verlag, Berlin, 1996).

[15] J. Anandan and Y. Aharanov, Phys. Rev. Lett. 65, 1697 (1990).

[16] S. L. Adler, "Statistical Dynamics of Global Unitary Invariant Matrix Models as Pre-Quantum Mechanics", Appendix I, hep-th/0206120.

[17] L. Diósi, private communication.

[18] N. Gisin, Phys. Rev. Lett. 52, 1657 (1984); L. Diósi, Phys. Lett. A 129, 419 (1988). For recent discussions of the imaginary versus real noise equations, see S. L. Adler and L. P. Horwitz, J. Math. Phys. 41, 2485 (2000), and S. L. Adler, Ref. [16], Sec. 5F.

[19] See e.g. P. Facchi and S. Pascazio, Ref. [14].

[20] I. I. Rabi, Phys. Rev. 51, 652 (1937); I. I. Rabi, N. F. Ramsey, and J. Schwinger, Rev. Mod. Phys. 26, 167 (1954); R. P. Feynman, F. L. Vernon, Jr., and R. W. Hellwarth, J. Appl. Phys. 28, 49 (1957).

[21] W. M. Itano, D. J. Heinzen, J. J. Bollinger, and D. J. Wineland, Phys. Rev. A 41, 2295 (1990).

[22] R. J. Cook, Phys. Scr. T 21, 49 (1988). 
[23] L. P. Hughston, private communication. For a textbook discussion, see L. Arnold, "Stochastic Differential Equations: Theory and Applications," Sec. 8.4 (Wiley-Interscience, New York, 1974). I wish to thank L. Diósi for bringing this reference to my attention. 